\title{
Dimensiones del espacio público y su importancia en el ámbito urbano*
}

\author{
Eduardo Julio Garriz, ${ }^{1}$ Romina Valeria Schroeder² \\ Universidad Nacional del Sur (Argentina)
}

Recibido: agosto 30 de 2014 - Revisado: noviembre 20 de 2014 - Aceptado: diciembre 8 de 2014

Referencia formato APA: Garriz, E. J., \& Schroder, R. V. (2014). Dimensiones del espacio público y su importancia en el ámbito urbano. Revista Cientifica Guillermo de Ockham, 12(2), 25-30.

\section{Resumen}

El presente artículo de reflexión diferencia las dimensiones que forman parte del espacio público, tratándolas de forma individual sin dejar de reconocer la profunda complementariedad e interdependencia que muchas veces no permite una identificación clara. A través de la revisión bibliográfica, se ha tratado de abordar el siguiente interrogante: ¿cómo responde el espacio público a una movilidad permanente producto de la cambiante relación sociedad-espaciocultura? Se ha procurado desagregar las dimensiones y diferenciarlas de manera más específica sin perder la condición de totalidad. Se entiende que no es un tema cerrado, sino que pretende estimular el debate sobre el espacio público.

Palabras clave: Espacio urbano, espacio público, transformaciones urbanas, nuevas centralidades

\section{Dimensions of public space and its importance in urban areas}

\section{Abstract}

This article deals with public space dimensions. They have been treated individually despite recognizing the profound complementarity and interdependence which often does not allow a clear identification. In this sense, there has been a literature review which has arisen new questions related to how public space responds to a permanent mobility due to changes in society-space-culture relationship. Therefore, efforts have been made to disaggregate dimensions and, specifically, not losing the integrated perspective. It is understood that it is not a closed issue, but instead is intended to stimulate discussion about public space.

Keywords: Urban space, public space, urban transformations, new centralities

* Este artículo de revisión es producto del proyecto de investigación Lógicas y tendencias en la expansión urbana y residencial de ciudades medias. El caso de Bahía Blanca a partir de 1990, con financiamiento de la Secretaría de Ciencia y Tecnología de la Universidad Nacional del Sur, Buenos Aires, Argentina. Director: Eduardo Julio Garriz. Fecha de inicio: enero de 2013. Fecha de finalización: diciembre de 2016.

1. Licenciado en Geografía. Profesor adjunto con dedicación exclusiva de la Universidad Nacional del Sur, Departamento de Geografía y Turismo. Bahía Blanca, Argentina. Dirección postal: 12 de octubre y San Juan, piso 4º gabinete 9, código postal (8000) Bahía Blanca, Buenos Aires, Argentina. E-mail: ejgarriz@uns.edu.ar

2. Doctora en Geografía. Licenciada en Turismo. E-mail: roschroeder@hotmail.com romina.schroeder@uns.edu.ar 


\section{Introducción}

Al considerar la ciudad como una construcción social, se debe tener en cuenta que el espacio urbano es producto de la materialización que los distintos agentes intervinientes producen, aspecto que se traduce en la espacialización de las acciones que dan lugar a las diferenciaciones socioespaciales que configuran la ciudad. Se puede destacar que "con frecuencia, los términos más usuales [...], cuando son mirados de cerca, son los más problemáticos, es decir, aquellos cuyos efectos sociales alcanzan una mayor complejidad" (Castells, 1971, p. 27), como es el caso del espacio público.

Jordi Borja se pregunta sobre el significado de la ciudad y su respuesta encierra una aparente simpleza. Este urbanista define la ciudad como "un lugar con mucha gente. Un espacio público, abierto y protegido [...] Una concentración de puntos de encuentro" (Borja, 2001, p. 391). Para este autor, la ciudad es un espacio público generalizado que pretende generar cohesión social e intercambios, pero que cada vez se encuentra atravesado por situaciones conflictivas y escenarios de desigualdades crecientes (Borja, 2003).

Precisamente, al ser el espacio público una parte sustancial de la ciudad, es un reflejo de sus contradicciones. Un importante número de aportes han tratado diferentes aspectos que atañen al espacio público y han intentado captar la compleja realidad presente en la ciudad (BelletSanfeliu, 2008; 2009; Body-Gendrot, 2008; Borja, 2005; Carrión, 2001; Formiga, 2007; Klaus, 2004; López de Lucio, 2000; 2013; Salcedo-Hansen, 2002).

De esta manera, la diversidad del espacio público permite establecer variaciones tipológicas, espacios de posibilidades, usos y acciones múltiples.

El espacio público es multidimensional y puede definirse de muchas formas según pongamos nuestra mirada en sus formas (espacio libre, espacio abierto, espacios transición), en su naturaleza (régimen de propiedad, tipo de gestión), en los usos y funciones que allí se desarrollan (espacio para el colectivo, común, compartido), o en el tipo de relaciones que se establecen (espacio de la presentación y representación, democracia, protesta, fiesta, etc.). Espacios con dimensiones muy diversas pero casi todas ellas relacionadas con un aspecto: el lugar de la expresión y representación social, civil y colectiva, el espacio democrático por excelencia, el espacio común (Bellet-Sanfeliu, 2009, p. 1).

Este artículo tiene como propósito plantear una reflexión sobre el espacio público y sus dimensiones sociales, mencionar algunas tipologías existentes y considerar algunas problemáticas de cobertura y accesibilidad que les atañen. En este texto el espacio público se entiende como una parte indisociable del entramado que conforma el espacio urbano y en el que se destacan distintas dimensiones que determinan su importancia como espacio de vida individual y colectiva, además de constituirse como un espacio asociado a la convivencia y generación de conflictos.

El espacio público es un concepto propio del urbanismo que a veces se confunde (erróneamente) con espacios verdes, equipamientos o sistema viario, pero que también es utilizado en filosofía política como lugar de representación y de expresión colectiva de la sociedad (Borja, 2005, p. 21).

En este sentido, los espacios públicos deben ser tratados de manera interdisciplinaria y multifuncional y las distintas dimensiones abordadas de manera individual, a fin de reconocer sus cualidades y su nivel de integración.

\section{El espacio público y sus dimensiones: Una aproximación desde lo conceptual}

Desde el punto de vista conceptual, se entiende que el espacio público responde a ciertas dimensiones -determinadas por algunos autores con algún grado de similitud-que permiten su cualificación. Bellet-Sanfeliu (2009) identifica aspectos como las formas que adquieren los espacios, los usos, las funciones y las relaciones y destaca las siguientes dimensiones: colectiva y cívica; simbólica y representativa; dinámico-funcional y la físico-urbanística. Para el presente trabajo, se considera estas dimensiones como válidas, pero pueden desagregarse aún más. Con la finalidad de ampliar los puntos de debate, se plantean las siguientes dimensiones:

\section{Dimensión físico territorial y urbanística}

Se considera que el espacio urbano (que no es neutral ni homogéneo), permite a partir de su condición física, una fuerte diferenciación por sus rasgos naturales, su emplazamiento, la calidad de su entorno y su accesibilidad. Durante el proceso de crecimiento y consolidación urbana, los espacios públicos adquieren valor al convertirse en territorios particularizados, producto de las intervenciones urbanísticas que van definiendo, a su vez, actividades y usos.

En este sentido, se destaca una importante diferencia funcional entre los requerimientos espaciales de una calle, una plaza o una plazoleta y un parque, el cual debe contar con mayor superficie y estar preparado para un cúmulo de 
actividades orientadas a una cantidad variable de usuarios. Cabe indicar, además, que no todas las ciudades cuentan con ambientes naturales, como una reserva, un frente de agua, una zona costera o un relieve montañoso; espacios estos más complejos que requieren buena accesibilidad, pues su área de influencia puede alcanzar el nivel regional.

En esta dimensión, es común la referencia a un territorio cuyas condiciones son ser visible, ser accesible para todos y tener un marcado carácter de centralidad. Por su parte Bellet-Sanfeliu (2009) considera al espacio público como aquel que es abierto a todos, accesible y multifuncional.

Respecto a la dimensión física del espacio, entendemos que si bien puede haber una estrecha relación con las condiciones del espacio natural -como elemento que otorga identidad y al mismo tiempo resultar limitante-, también puede ser más amplio e incorporar aquellos elementos que no permiten el pleno acceso al espacio, como barreras físicas, simbólicas, psicológicas y normativas, entre otras.

Si bien son elementos destacables en términos de dimensión física, las características del espacio son centrales para concretar un tipo de espacio público. No obstante, estos deben ser de conocimiento de la sociedad, de acceso libre para los habitantes y de carácter polifuncional para satisfacer las múltiples necesidades de sus usuarios y potenciales visitantes.

En este proceso de construcción del espacio urbano y dados su impacto urbanístico y los cambios en el territorio, cabe mencionar los espacios que cumplen con la condición de uso público aunque sean de naturaleza privada. Un aspecto característico del espacio público actual tiene relación, precisamente con "El surgimiento de nuevos espacios privados que asumimos como públicos y se encuentran cada vez más bajo la propiedad y el control privados" (Galindo-Muñoz, 2009, p. 13).

Se trata de galerías en cualquiera de sus tipos: malls, shopping centers y otros espacios similares que cumplen con la premisa de ser propiedad privada y de uso público; estructuras creadas que se adaptan a la ciudad, pero con un fuerte posicionamiento hacia la accesibilidad. Su finalidad está asociada al consumo y al esparcimiento y su condición de espacio público es limitada. Dicho de otra manera, pueden ser lugares de encuentro a partir de una apropiación no planeada, pero su carácter de propiedad privada genera dudas acerca de posibles condicionamientos en el acceso que potenciales usuarios puedan llegar a tener.

También debe ser objeto de análisis los espacios de interconexión que crean y combinan la condición natural con nuevas formas vinculadas al uso del espacio. Al respecto, resulta interesante observar cómo se entrelazan espacios diferenciados, a saber, plazas, parques, parques lineales y sendas, con equipamientos específicos (pistas de salud, juegos para niños y particularizados para personas con limitaciones motrices o neurológicas). Este espacio, dada su complejidad, no tiene una tipología que lo identifique claramente y aquellas que se utilizan para tipificarlas son parcialmente válidas, por lo que requiere un nuevo encuadre tipológico, puesto que el valor del conjunto supera al de las partes. Asimismo, debe agregarse la producción de conflictos al no estar identificados de manera clara su utilización por los múltiples usuarios y para diversas actividades (ciclistas, peatones, skaters y runners, entre otros).

\section{Dimensión jurídico-política}

Esta última dimensión muestra la manera como se interconectan las dimensiones, la importancia del espacio físico y las actividades que configuran nuevos escenarios de sociabilidad, producto de los profundos cambios en el modo de vida y en las formas de recreación que se observan en las dinámicas urbano-territoriales.

En esta esfera, se entiende el espacio público como una "categoría puente" en la que se conectan y entrecruzan diversas dimensiones de la ciudad, especialmente lo cívico y lo político. Cuando se efectúa una cronología referida a los espacios públicos que toma fundamentalmente su esencia, se puede afirmar que es "más que un simple lugar de propiedad compartida que se utiliza para desplazarse y para relacionarse" (López de Lucio 2013, p.19). Existe una dimensión política vinculada al diálogo con la administración pública, propietaria en unos casos y en otros faculta el dominio del suelo que garantiza su uso y fija las condiciones de utilización e instalación de actividades (Borja, 1998). Lo anterior da lugar, por un lado, a una función social pública y por el otro, a un carácter jurídico del dominio público. Es decir, esta dimensión asume que

El espacio público moderno proviene de la separación formal (legal) entre la propiedad privada urbana (expresada en el catastro y vinculada normalmente al derecho de edificar) y la propiedad pública (o dominio público por subrogación normativa o por adquisición de derecho mediante cesión) que normalmente supone reservar este suelo libre de construcciones (excepto equipamientos colectivos y servicios públicos) y cuyo destino son usos sociales característicos de la vida urbana (Borja, 1998, p. 13).

A partir de la gestión de su administración, la ciudad debe garantizar espacios públicos y de expresión de los derechos cívicos de los ciudadanos, que introduzcan 
mecanismos de integración y posibiliten una mayor calidad de vida en residentes y potenciales visitantes. Si bien se considera necesaria una legislación que se base en la protección, el mantenimiento, el cuidado y el resguardo del espacio público como bien social, lo que sí debe quedar claro es que el concepto de espacio público debe ser flexible en cuanto a que la administración pública debe estar atenta a cómo este espacio se reproduce, muta y se adapta y en qué medida las normas acompañan este proceso de cambio.

\section{Dimensión social}

Vinculada con lo anterior, esta dimensión reivindica el sentido de apropiación del espacio público por parte de los habitantes de una comunidad social y ello implica la importancia que le otorgan en cuanto a su valoración, en cuanto a las normas que los resguarden y, en definitiva, en cuanto a la manera como lo asumen como propio. No obstante lo anterior, hay diferencias importantes entre los distintos grupos sociales y de ellas derivan los conflictos que se generan en el uso de los espacios públicos.

Los diversos espacios son atravesados por acciones que pueden resultar funcionales o disfuncionales para las pautas imperantes, situación que persistirá hasta que se modifiquen por la aceptación de los nuevos usos y se determinen las pautas de funcionamiento para el beneficio común. Es en el espacio público donde la vida colectiva se realiza y se expresa. La calle se conquista por grupos que reivindican, festejan o reclaman; en definitiva, se pronuncian, y convierten -en algunos casos- ciertos lugares en verdaderos hitos para el imaginario colectivo.

La ciudad es permeable a los cambios; por ello, en su permanente e imperceptible devenir su dinámica se impone, se modifica su estructura y sus prácticas cambian. Por tal razón, muchos espacios adquieren el valor de público no por moda, sino como apropiación espontánea de la sociedad. Así, estos cambios sociales son resultado de acciones individuales o de determinados grupos -que pueden incluir la categoría de emergentes- que se apropian de ciertos lugares y los asumen como propios, pero a su vez esto espacios son parte del colectivo. No es de extrañar que este proceso se repita en un ciclo de renovación cuasi permanente.

Aquí, cualquiera de los tipos de espacio público vive una condición múltiple de cierta ambigüedad: entre el anonimato y la necesidad de socialización. Quizás, este sea uno de los aspectos más ricos, pero más difíciles de cuantificar y visualizar, pues cada persona lo percibe a su manera, lo vive con intensidad diferente y lo frecuenta conforme con su edad, su grupo de referencia y las actividades que le son propias.

Los espacios públicos, como base de cualquier forma verdadera de integración social, deben ser escenarios de convivencia donde no se obligue a nadie a justificar su origen, su condición social o su idiosincrasia. Borja (2005) menciona que el espacio público es concebido como instrumento de redistribución social, de cohesión comunitaria, de autoestima colectiva. Sin embargo, en él se presentan distintas relaciones de poder vinculadas con el sexo, la edad y las dinámicas que se establecen, que pugnan por el control de los espacios y que muchas veces resultan en un impedimento para su pleno y equitativo uso.

\section{Dimensión cultural y simbólica}

Si bien el espacio público debe verse como una categoría amplia, cuyo conjunto en general excede las tipologías establecidas como básicas (pues su esencia es el todo), el peso que tiene en la comunidad, su herencia histórica y su valor patrimonial, crean lugares y estos se incorporan a manera de imaginarios individuales y colectivos.

La cultura se representa en lo material y lo inmaterial, según requerimientos y pautas culturales de los grupos, por aceptación consciente o inconsciente de la sociedad. De esta forma, se van sucediendo la ocupación de los espacios y sus usos y estos adquieren importancia por parte de los habitantes de la ciudad.

Lo anterior puede hacerse visible en un área como el casco fundacional $\mathrm{u}$ otras que la sociedad asuma como propias. Son expresiones espontáneas en las que la simbiosis habitante-lugar se materializa en aspectos que reconocemos como identidad simbólica del lugar. En el caso de los jóvenes, por ejemplo, es visible la forma como utilizan un espacio que podría ser para la comunicación personal o la recreación pasiva o activa, para convertirlo en un ámbito de comunicación interpersonal y a distancia, dado que la provisión de wifi en ciertos espacios públicos lo permite; es un espacio virtual dentro de un espacio que se concibe para el disfrute. Lo anterior replantea la condición del espacio público ante el avance tecnológico, las nuevas formas de comunicación y la interrelación personal.

\section{Dimensión económica}

En esta dimensión, pueden establecerse distintas formas de apropiación del espacio público frente a un uso específico: en este caso, la actividad económica. Posiblemente, la calle sea la más generalizada y la que ofrece mayor diversidad y posibilidades, dadas su extensión e intersecciones, situación que favorece algunas actividades, contrariamente 
a lo que brinda una plaza, un parque o cualquier otro espacio que se encuentre limitado a días o eventos.

Se distinguen distintos tipos de apropiación respecto al uso que se le da a la calle, como la venta de diarios, paradas de taxis y otros puestos de venta (suvenires, artesanías, comidas rápidas, floristas, vendedores de refrescos). Hay también malabaristas que utilizan la calle para demostrar sus habilidades y limpiacoches que seleccionan los lugares de tránsito intenso y lento (semáforos). En gran medida, estas actividades emergen de la necesidad y se encuentran vinculadas a la precariedad y subsistencia; son la posibilidad de tener una actividad laboral bajo un trabajo informal. En definitiva, la calle es su refugio, su posibilidad de ser reconocidos y ser legibles a una sociedad que en muchos casos los ignora o considera marginales.

\section{Dimensión de movilidad y apropiación}

La ciudad, como espacio donde convergen flujos, lleva implícita esta dimensión. La movilidad, el uso y la apropiación pueden vincularse a la dinámica que modifica el territorio y se ponen de manifiesto tanto en los diferentes espacios como en los usos que se les dan. Se distinguen aquí, los siguientes:

a. Espacios sin consenso, cuya creación se hizo sin consulta a la comunidad y el resultado fue la no utilización o subutilización.

b. Espacios démodé, cuya finalidad varía según los cambios en las pautas culturales, un ejemplo de ellos son los jardines zoológicos.

c. Espacios de nueva valoración, asociados al reemplazo de lugares que tenían un estatus determinado para la sociedad y son cambiados -en muchos casos- por nuevos lugares que congregan a la comunidad por aceptación directa y no por determinación acordada.

d. Espacios de cambio espontáneo, son aquellas áreas de la ciudad que los habitantes adoptan como espacios públicos de manera natural, como son los espacios de borde urbano.

En esta dimensión se pretende demostrar cómo se interrelacionan la sociedad, las pautas culturales, las decisiones de la gestión y el valor que tiene el consenso en cuanto a la determinación, el uso y los cambios que requiere el espacio público.

\section{Dimensión virtual}

Una lectura más determinante respecto de la ciudad, agrega, necesariamente, la dimensión de la virtualidad y su influencia en los cambios experimentados por la ella. En los últimos años hemos asistido a la emergencia de un tipo de ciudad completamente nueva, una ciudad sin un lugar asociado a ella (Sorkin, 2004). En la dimensión virtual los lugares disipan su significado geográfico, histórico y cultural y quedan integrados como lugares funcionales productores de un espacio de flujos que sustituye el espacio físico (Castells, 1997).

Quizás, esta asociación con lo físico como punto referencial de algo (llámese encuentros, eventos, manifestaciones sociales) no solo afecta al lugar, sino que también se convierte en un asunto de lugares, de cambios generacionales y de movilidades que ya no tienen la referencia de un hito o de un valor comunitario.

Como aspecto final, debe tenerse en cuenta cómo las nuevas tecnologías y los modos de vida generan cambios y transformaciones tanto a nivel de ciudad como de espacio público. Es así, que las transformaciones que ha experimentado la misma ciudad, la sociedad y la urbanidad en general, han tenido un claro impacto en el espacio público.

\section{A modo de conclusión}

El espacio público implica distintas dimensiones que, en su conjunto, lo definen y debería ser tratado como un concepto complejo que va más allá de la antinomia público-privado. En tal sentido, la propuesta de este artículo ha sido desagregar las dimensiones para identificar las particularidades presentes en cada una de ellas y si bien las reflexiones presentadas aquí resultan insuficientes para arribar a conclusiones concretas, es posible observar una complementariedad y una profunda interrelación entre las dimensiones del espacio público e inferir, además, la razón del solapamiento y la dificultad para su análisis en los diversos abordajes estudiados.

La ciudad en su conjunto y las dinámicas que la reestructuran, requieren planteamientos permanentes sobre el lugar que ocupan los espacios públicos dentro de las problemáticas urbanas. Por ende, las dimensiones que hemos tratado merecen una indagación continua a fin de incorporarlas al estudio del espacio público y mantener esta idea de integralidad conceptual.

Debe destacarse el hecho físico como expresión material que diferencia los espacios tanto a nivel morfológico como funcional y genera diversos escenarios de posibilidades, uso y acciones. Pero, también es necesario tener en cuenta cómo estos espacios son valorados, percibidos y apropiados por los distintos sectores que componen la sociedad, lo 
cual genera interrogantes vinculados a la relación sociedadespacio-cultura en el devenir temporal: ¿qué es lo que la sociedad interpreta por espacio público?, ¿cómo influyen las pautas culturales sobre dichos espacios?, ¿¿n qué medida repercutirá la influencia de las nuevas tecnologías en la configuración y usos de los espacios públicos?

Tal situación merecería considerar el establecimiento de un estado de crisis en el espacio público, al meditar acerca de si estos se comportarán como meros soportes físicos o elementos relictuales de una cultura que los asumía como espacios de relación y convivencia.

De esta manera, los términos visibilidad, accesibilidad y apropiación, son vitales para interpretar los cambios en el espacio público. Vinculado con lo anterior, surge la pregunta sobre la necesidad de trabajar -a partir de la dimensión jurídica- sobre códigos de convivencia pública. En este sentido, debe estar presente el diálogo y el trabajo conjunto en la gestión y la sociedad, apoyados en las instituciones intermedias y por expertos que valoricen y busquen concientizar, desde las etapas más tempranas de la formación de la persona, acerca del verdadero significado de espacio público y no simplemente en términos de cantidad y tipologías, todo con miras mejorar la calidad de vida. Esta situación requiere un proyecto de ciudad que integre el espacio público como parte indisociable del espacio urbano.

\section{Referencias}

Bellet-Sanfeliu, C. (2008). El espacio público y la nueva urbanidad: Reflexiones sobre el caso de las ciudades intermedias. En J. M. Llop (Eds. et al.), Ciudades en re-construcción: Necesidades sociales, transformación y mejora de barrios (pp. 207-230). Barcelona: Diputación de Barcelona.

Bellet-Sanfeliu, C. (Noviembre, 2009). Reflexiones sobre el espacio público. El caso de las ciudades intermedias. Trabajo presentado en el IV Seminario de la Facultad de Arquitectura y Diseño de la Universidad de los Andes. Mérida, Venezuela. Recuperado de http://goo.gl/2CE9ma

Body-Gendrot, S. (Septiembre, 2008). Grandes evoluciones sociales en las áreas metropolitanas. Trabajo presentado en el segundo seminario organizado por el Pla Estratègic Metropolità de Barcelona. Espańa. Recuperado de http:// goo.gl/8VAKl5
Borja, J. (1998). Ciudadanía y espacio público. En J. Borja (autor), Laberintos urbanos en América Latina (pp. 9-26). Recuperado de http://goo.gl/a6LZ8b

Borja, J. (2001). La ciudad del deseo. En F. Carrión (Ed.), La ciudad construida. Urbanismo en América Latina (pp. 391396). Ecuador: FLACSO.

Borja, J. (2003). La ciudad conquistada. Madrid: Alianza Editorial.

Borja, J. (Abril, 2005). Revolución y contrarrevolución en la ciudad global. Revista Bibliográfica de Geografia y Ciencias Sociales. Recuperado de http://www.ub.edu/geocrit/b3w578.htm

Carrión, F. (2001). Las nuevas tendencias de la urbanización en América Latina. En F. Carrión (autor.), La ciudad construida. Urbanismo en América Latina. Ecuador: FLACSO.

Castells, M. (2014). El mito de la sociedad urbana. Revista Latinoamericana de Estudios Urbanos Regionales, 1(3), 27-41.

Castells, M. (1997). El surgimiento de la sociedad de redes. Madrid: Alianza.

Formiga, N. (2007). El derecho a la ciudad y la cuestión del espacio público: Experiencias en la ciudad de Bahía Blanca. Journal of Latin American Geography, 6(1), 174-196.

Galindo-Muñoz, O. (2009). Nuevas tipologías de espacio público en la construcción de la imagen competitiva de la ciudad de Medellin. Recuperado de http://goo.gl/qxoZ8i

Klaus, P. (2004). Urban Settings in the Competition among Cities. Recuperado de http://goo.gl/T7wn1l

López de Lucio, R. (2000). El espacio público en la ciudad europea: entre la crisis y las iniciativas de recuperación. Implicaciones para Latinoamérica. Revista Occidente, 230-231. Recuperado de http://goo.gl/lfDcoE

López de Lucio, R. (2013). Vivienda colectiva, espacio público y ciudad: Evolución y crisis en el diseño de tejidos residenciales 1860-2010. Buenos Aires: Nobuko.

Salcedo-Hansen, R. (Septiembre, 2002). El espacio público en el debate actual: Una reflexión crítica sobre el urbanismo postmoderno. Revista EURE (Santiago). 28(84). Recuperado de http://goo.gl/jeaujg

Sorkin, M. (Ed.). (2004). Variación sobre un parque temático: La nueva ciudad Americana y el fin del espacio público. Barcelona: Gustavo Gili. 